\title{
Retrograde Pulmonary Perfusion in Surgical Embolectomy for Massive Pulmonary Embolism
}

\author{
Won Ho Chang \\ Department of Thoracic and Cardiovascular Surgery, Soonchunhyang University Seoul Hospital, Seoul, Korea
}

\begin{abstract}
Mortality rate for pulmonary embolectomy in critically ill patients still ranges from $30 \%$ to $45 \%$. The causes of death in these patients are persistent pulmonary hypertension, pulmonary edema, and massive pulmonary hemorrhage. Residual thrombus and air trapping in peripheral pulmonary artery during pulmonary embolectomy can cause intractable right heart failure and persistent pulmonary hypertension. We report a successful extraction of residual thrombus and air bubbles during pulmonary embolectomy by retrograde pulmonary perfusion. Use of this technique could decrease morbidity and mortality from persistent right heart failure after pulmonary embolectomy in critically ill patients.
\end{abstract}

Keywords: Pulmonary embolism; Embolectomy; Thoracic surgery

\section{INTRODUCTION}

Surgical embolectomy for massive pulmonary embolism has been improved by judicious selection of patients, rapid diagnosis, and prompt surgery. However, mortality rates have been reported from $30 \%$ to $45 \%$ after surgical embolectomy for critically ill patients, and exceed $60 \%$ for cardiac arrest patients [1]. Emergency extracorporeal life support can be critical modality for life-saving when applied earlier for hemodynamically unstable patient.

Retrograde pulmonary perfusion using pulmonary vein was reported to relieve cyanoacrylate glue obstruction of the pulmonary artery in 1995 [2]. This technique facilitates the removal of residual thrombus and entrapped air from the peripheral branch of the pulmonary artery [1].

We describe retrograde pulmonary perfusion as an adjunct to surgical embolectomy for massive pulmonary embolism in a critically ill patient.

\section{CASE REPORT}

\section{Patient profile}

A 22-year-old male was transferred to the emergency room of
Soonchunhyang University Seoul Hospital from another hospital for treatment of cardiac arrest. He had received orthopedic surgery 2 days previously to lengthen both legs. He collapsed after complaining of severe chest pain. He had no medical history of venous thrombosis or thrombophilia. There was no response to conventional cardiopulmonary resuscitation so we performed venoarterial extracorporeal life support by femoral cannulation. After initiation of extracorporeal circulation, cardiac activity was recovered. On transthoracic echocardiography, the right ventricle was distended and systolic function was decreased. Chest computed tomography revealed a massive pulmonary embolism (Fig. 1). Persistent hypotension and right ventricular dysfunction were evident. The patient had received the recent orthopedic surgery, multiple rib fractures resulted from prolonged cardiopulmonary resuscitation, and extracorporeal life support by femoral artery cannulation, thus a risk of bleeding might be high for a thrombolytic therapy. We decided to perform emergency surgical embolectomy.

\section{Surgical technique}

The pulmonary embolectomy was performed using moderate hypothermia cardiopulmonary bypass with bicaval cannulations. During surgery, the peripheral extracorporeal life support was 


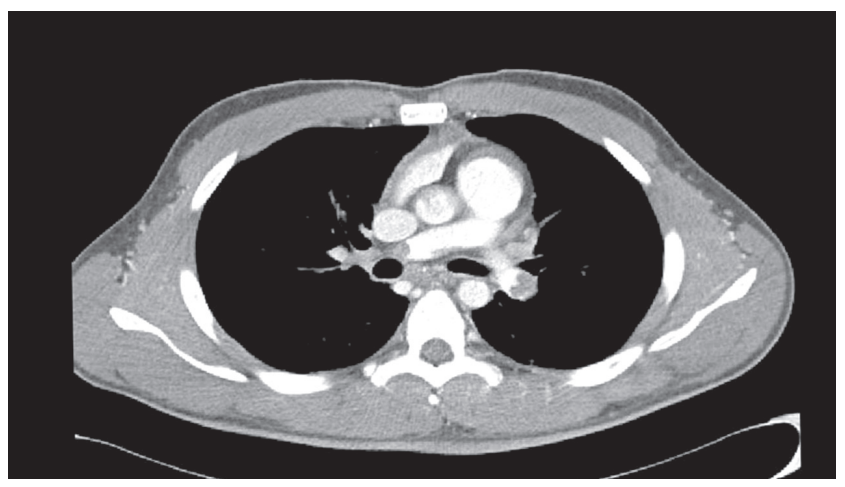

Fig. 1. Chest computed tomography showing acute and massive pulmonary thromboembolism in pulmonary arterial trunk and both main pulmonary arteries.

maintained with minimal flow less than $500 \mathrm{~mL} / \mathrm{min}$. The arterial line of extracorporeal circuit was connected to a Y connector and extended with additional arterial line, which was clamped. The other arterial line was connected with arterial cannula into the ascending aorta. A vent cannula was inserted into the left ventricle through a purse-string suture placed on the right upper pulmonary vein. After initiation of cardiopulmonary bypass, the patient was cooled until core temperature dropped under $18^{\circ} \mathrm{C}$. The aorta was cross-clamped and the cardioplegic solution was infused into the aortic root. Under total circulatory arrest, a longitudinal incision was made on the pulmonary artery trunk and was extended into the proximal right and left pulmonary artery branches. The thrombus was extracted using forceps. Residual thrombus in pulmonary artery branches could not be removed satisfactorily. The clamped additional arterial line was connected with a vent cannula and the cannula was repositioned into the left atrium (Fig. 2). While the pulmonary artery was still open, the operator grasped the left ventricle to prevent distension during retrograde pulmonary perfusion. The clamp of an additional arterial line into the left atrial cannula was released. Blood filled the left atrium and began flowing into the pulmonary artery in a retrograde fashion. The lungs were inflated during retrograde pulmonary perfusion to facilitate the elimination of residual thrombus and air bubbles in the distal branches of the pulmonary artery. The residual clots were aspirated and pulmonary arteriotomy was closed (Fig. 3). The aorta was declamped and the flow of peripheral extracorporeal life support was increased up to $3.5 \mathrm{~L} / \mathrm{min}$. The patient was weaned from cardiopulmonary bypass. Cardiopulmonary bypass time was 50 minutes including 12 minutes total circulatory arrest time.

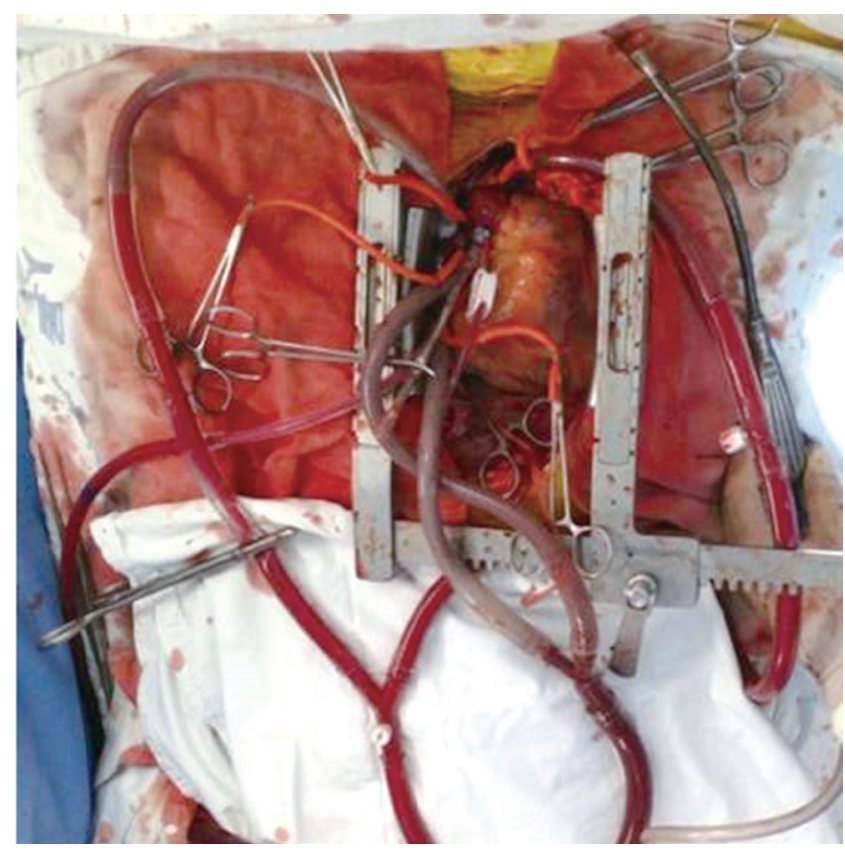

Fig. 2. Extracorporeal circuit set up for retrograde pulmonary perfusion. Vent cannula connected to the arterial line by Y-connector was inserted into left atrium.

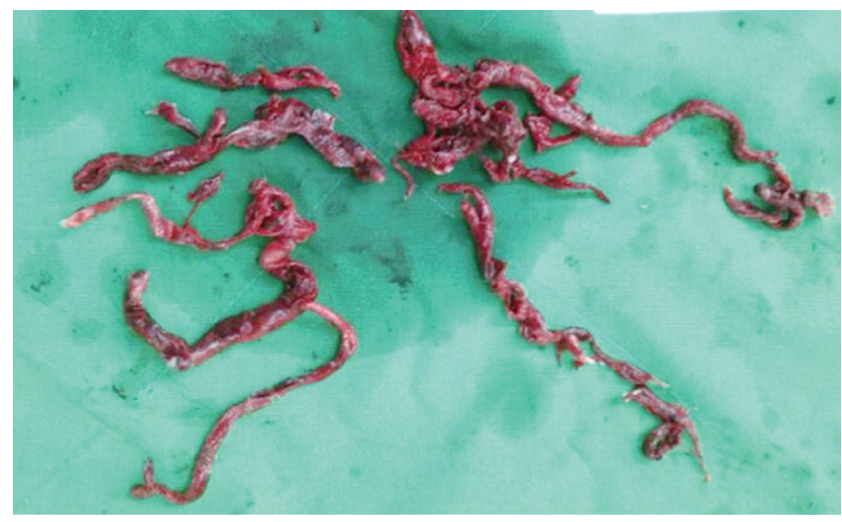

Fig. 3. Large thrombus in main pulmonary artery and residual thrombus in peripheral pulmonary arteries were extracted by forceps and retrograde pulmonary perfusion.

At the intensive care unit, milrinone was infused for treatment of right ventricular dysfunction, heparin was infused for anticoagulation, right ventricular and respiratory function were recovered, and extracorporeal life support could be weaned successfully at postoperation day 3. Patient had cognitive dysfunction result from hypoxic encephalopathy during cardiac arrest. He was transferred to rehabilitation department for integrated rehabilitation. 


\section{DISCUSSION}

The incidence of pulmonary embolism is rare but the mortality rate is greater than $15 \%$ within the first 3 months after the diagnosis. Patients usually appear to be stable for 12-48 hours, with venous thrombus formation occurring spontaneously. However, sudden collapse with hypotension and cardiac arrest can occur. In acute pulmonary embolism, right ventricular dysfunction is an important prognostic marker for high risk patients and poor outcomes [3]. Extracorporeal cardiopulmonary resuscitation using veno-arterial extracorporeal circulation is a crucial procedure for hemodynamic deteriorated and cardiac arrest patients due to a massive pulmonary embolism. The procedure allows the right ventricle function to recover [4].

Patients who undergo pulmonary embolectomy most often have experienced right heart failure secondary to pulmonary hypertension, pulmonary edema, and pulmonary hemorrhage. Residual thrombotic material in the distal pulmonary arterial tree is an important cause of pulmonary hypertension. Suction, forceps, and Fogarty catheters are used for extraction of remnant clots through extended pulmonary arteriotomy with manual compression of the lungs. Blind instrumentation for extraction of invisible clot is very dangerous, and can damage distal pulmonary vessels. Endobronchial and parenchymal bleeding could result from direct mechanical injury of the pulmonary arterial wall. Adverse and fatal effects of air embolism in the pulmonary artery during pulmonary embolectomy have been described. Pulmonary air embolism can release endothelium-derived cytokine that can cause pulmonary hypertension [5].

Retrograde pulmonary perfusion was first used for flushing of a cyanoacrylate glue obstruction from the distal pulmonary artery after embolization of cerebral arteriovenous malformation in a 3-year-boy [2]. In a series of 21 consecutive critically ill patients receiving retrograde pulmonary perfusion while performing pulmonary embolectomy, there were no deaths or major postoperative complications [1].

The complete removal of residual thrombus from distal branches of pulmonary artery was facilitated by retrograde pulmonary perfusion. By filling the pulmonary arterial tree with blood after pulmonary embolectomy, retrograde pulmonary perfusion eliminated entrapped air and its' negative effect.

\section{ACKNOWLEDGMENTS}

Informed consent: Written informed consent was obtained from the patient for publication of this manuscript and any accompanying images.

\section{REFERENCES}

1. Spagnolo S, Grasso MA, Tesler UF. Retrograde pulmonary perfusion improves results in pulmonary embolectomy for massive pulmonary embolism. Tex Heart Inst J 2006;33:473-6.

2. John LC, Awad WI, Anderson DR. Retrograde pulmonary embolectomy by flushing of the pulmonary veins. Ann Thorac Surg 1995;60:1404-6.

3. Goldhaber SZ, Elliott CG. Acute pulmonary embolism: part I: epidemiology, pathophysiology, and diagnosis. Circulation 2003;108:2726-9.

4. Sistino JJ, Blackwell M, Crumbley AJ. Transport on emergency bypass for pulmonary embolism followed by surgical repair using retrograde pulmonary perfusion: a case report. Perfusion 2004;19:385-7.

5. Kucher N, Goldhaber SZ. Management of massive pulmonary embolism. Circulation 2005;112:e28-32. 